\title{
Performance characteristics of high resolution Compton telescopes
}

\author{
S. E. Boggs ${ }^{1}$ and P. Jean ${ }^{2}$ \\ 1 Department of Physics, University of California, Berkeley, CA, 94720 USA \\ 2 Centre d'Étude Spatiale des Rayonnements, UPS-CNRS, Toulouse, France \\ e-mail: Pierre. Jean@cesr.fr
}

Received 23 April 2001 / Accepted 17 July 2001

\begin{abstract}
To build upon the goals of the upcoming INTEGRAL mission, the next generation soft $\gamma$-ray $(0.2-20 \mathrm{MeV})$ observatory will require improved sensitivity to continuum and nuclear line emission, while maintaining high spectroscopy to resolve Doppler shifts and broadenings. We present the simulated performance of two germanium Compton telescope (GCT) designs in high Earth orbit conditions. The goal of this work is both to determine realistic sensitivities for the GCTs, as well as determine the geometry which best takes advantage of the spectral, imaging, and stopping power of germanium detectors. We find that a novel Compact design optimizes the sensitivity to both nuclear line and continuum sources, with an effective factor of 2.0 improvement over a Classical design. The Compact GCT would provide an order of magnitude improvement in sensitivity over INTEGRAL.
\end{abstract}

Key words. gamma rays: observations - telescopes - techniques: spectroscopic - techniques: image processing instrumentation: detectors

\section{Introduction}

The Compton Gamma-Ray Observatory (CGRO) made dramatic achievements in the study of soft gamma-ray sources, especially in the study of ${ }^{26} \mathrm{Al}$ $(1.809 \mathrm{MeV})$ and ${ }^{44} \mathrm{Ti}(1.157 \mathrm{MeV})$ with COMPTEL (Schönfelder et al. 1993), and positron annihilation radiation $(0.511 \mathrm{MeV})$ with OSSE (Johnson et al. 1993). The INTERnational Gamma-Ray Astrophysics Laboratory (INTEGRAL), scheduled for launch in April 2002, will build upon these discoveries with improved sensitivities and much higher spectral resolution with SPI (Jean et al. 2000), and angular resolution with IBIS (Ubertini et al. 2000).

Looking toward the future, the next generation soft gamma-ray observatory will have to build upon the scientific strengths of INTEGRAL. For the study of nuclear line emission, this instrument will require significantly improved sensitivity while maintaining very high spectral resolution. A high priority goal will be the systematic study of ${ }^{56} \mathrm{Co}(0.847 \mathrm{MeV})$ lines, Doppler broadened $5000-10000 \mathrm{~km} \mathrm{~s}^{-1}$, from type Ia supernova. This scientific goal ideally requires an instrument with

Send offprint requests to: S. E. Boggs,

e-mail: boggs@ssl. berkeley.edu broadened line sensitivities capable of measuring a handful of SN Ia per year, and spectral resolutions $\leq 1000 \mathrm{~km} \mathrm{~s}^{-1}$, or $\leq 0.3 \%$. Such a mission has been outlined in the NASA strategic plan as the Advanced Compton Telescope, and researchers are pursuing various instrument designs to reach these goals.

Combining the background rejection capabilities of COMPTEL and the spectral resolution of SPI, a number of researchers (Johnson et al. 1996; Jean et al. 1996a; Boggs 1998) have discussed the merits of a germanium Compton telescope (GCT). The development of Compton telescopes began in the 1970's (Schönfelder et al. 1973; Herzo et al. 1975; Lockwood et al. 1979), and culminated in the design and flight of COMPTEL. (See von Ballmoos et al. 1989, for a review of historical Compton telescope configurations.) In addition to GCTs, there is currently active development towards both silicon (Schopper et al. 2000; O'Neill et al. 2000; Kroeger et al. 2000) and liquid xenon (Aprile et al. 2000) instruments. Compton telescopes work on a well-known principle (Fig. 1): by measuring the positions and energies of the photon interactions the initial photon direction can be reconstructed to within an annulus on the sky using the Compton scatter formula. The uncertainty, or width, of this annulus depends on the spatial and spectral 

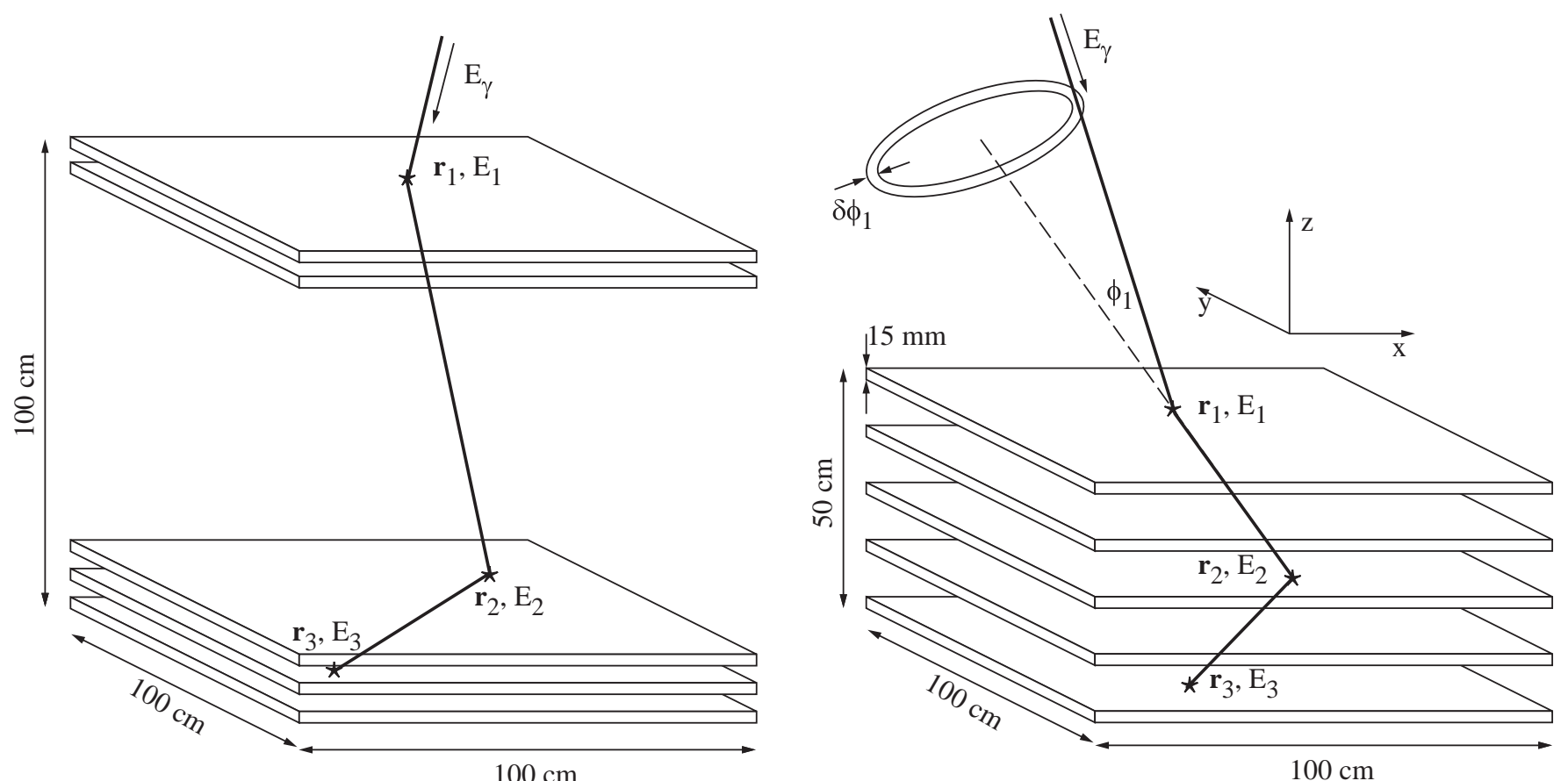

Fig. 1. The two GCT configurations analyzed in this work. The Classical configuration (left) is similar in concept to historical Compton telescope geometries as well as the GCT concept studied by Johnson et al. (1996). The Compact configuration (right) is designed to improve the effective area and FOV, using the same detector volume. In Compton telescopes the photon interaction locations, $r_{i}$, and energy depositions, $E_{i}$, allow reconstruction of the initial photon direction through the Compton scatter formula to within an annulus on the sky, $\phi_{1}$ (Paper I, and references therein).

resolution of the detectors, but also has a fundamental limit set by Doppler broadening due to Compton scattering off of bound electrons. Reconstruction of the event annulus requires that the first and second photon interaction locations in the instrument are spatially resolved, and their order properly determined.

Germanium detectors (GeDs) offer several critical advantages over other detector types for an Advanced Compton Telescope. GeDs have the advantage of large detector volumes $\left(\geq 75 \mathrm{~cm}^{3}\right)$, excellent uniformity, and good photon stopping power. The high spectral resolution $(0.2 \% F W H M$ at $1 \mathrm{MeV})$ would resolve Doppler shifts and broadenings of nuclear lines $\left(700 \mathrm{~km} \mathrm{~s}^{-1}\right.$ FWHM at $1 \mathrm{MeV}$ ). For example, such a spectral resolution is useful for measuring the velocity of radioactive elements in the ejecta of supernovae, providing unique information on the nucleosynthesis and dynamics of the explosion. The spectral resolution is also crucial for rejecting background events: the ability to discriminate and reject narrow internal background lines, characteristic $\beta^{-}$-decay photons, and $0.511 \mathrm{MeV}$ signatures from $\beta^{+}$-decays and pair-production events depends critically on the high spectral resolution.

Historical Compton telescope configurations make two assumptions about the events which do not generally hold in GCTs: (i) photons undergo a single Compton scatter in the converter, followed by photoelectric absorption in the absorber, and (ii) the time-of-flight (TOF) between the photon interactions is measured to determine their order. Measuring TOF has been especially critical in historical designs for rejecting background photons which originate from below the instrument. The expected event timing resolution in germanium detectors (>10 ns) is not adequate to determine the TOF for reasonable GCT configurations.

In a previous paper, we presented techniques for event reconstruction in GCTs (Boggs \& Jean 2000, hereafter Paper I). The goal of that paper was to describe, in detail, analysis techniques for reconstructing the event ordering in absence of a TOF measurement, as well as rejecting background. We defined a technique labeled Compton Kinematic Discrimination (CKD), which serves as a very powerful tool for determining the interaction ordering and rejecting background events (including the upward background component) in high resolution Compton telescopes. Such a technique had been previously discussed in context of liquid xenon telescopes by Aprile et al. (1993), and can also be used in silicon Compton telescopes. Also in Paper I we summarized additional techniques for rejecting $\beta^{-}$-decays, $\beta^{+}$-decays, and pair-production events. In a second paper (Boggs \& Jean 2001, hereafter Paper II) we presented in detail how the choice of event reconstruction techniques and event cuts affect the performance of a single GCT design, the Compact design presented in this paper. We summarize the results of Paper II below.

In this paper we present detailed analysis and comparison of the performance of two different GCT designs assuming a high Earth orbit (HEO). Our goal is to determine how the instrument geometry can affect the overall performance of the GCT. In particular, we are interested in determining the geometry which best utilizes the high 


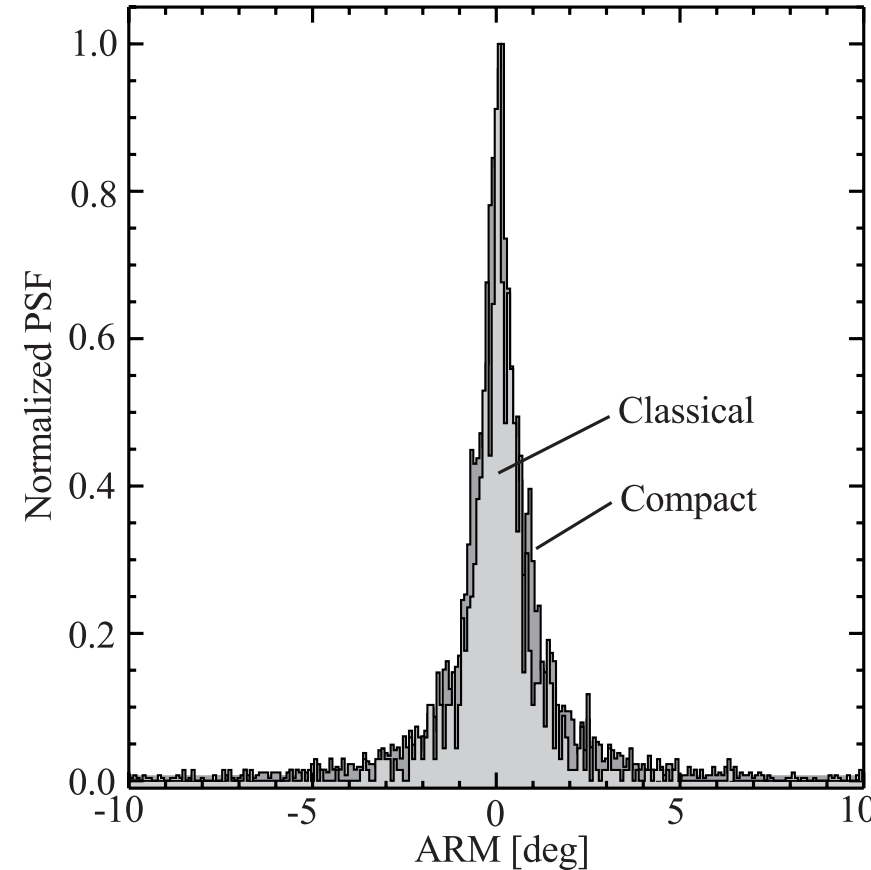

Fig. 2. The on-axis ARM distribution for $1 \mathrm{MeV}$ photons for the Classical and Compact geometries.

spectral and spatial resolution of the GeDs to optimize the sensitivity.

\section{Methods}

\subsection{Telescope simulations}

The two telescope configurations modeled in this study are presented in Fig. 1. Each instrument consists of five planar arrays of $15 \mathrm{~mm}$ thick germanium of area $100 \mathrm{~cm} \times 100 \mathrm{~cm}$. In reality each array would consist of separate smaller detectors $(\sim 7 \mathrm{~cm} \times 7 \mathrm{~cm})$ tiled to form the entire plane; however, the simulation performed here modeled each plane as a solid detector for simplicity. The $15 \mathrm{~mm}$ thickness has been chosen to optimize the Compton efficiency in the gamma-ray line energy range (between $0.2 \mathrm{MeV}$ and $1 \mathrm{MeV}$ ), and corresponds to a realistic GeD thickness. The Classical configuration is defined by two "converter" planes, and three "absorber" planes in a geometry similar to past Compton telescope designs, and to the GCT design studied in Johnson et al. (1996). The distance between the top face of the top converter detector plane and the top face of the bottom absorber detector plane was defined as $100 \mathrm{~cm}$, while the gap between detector planes within the converter or absorber was set at $2 \mathrm{~cm}$ (physically, it might be difficult to space detector planes any closer). In the Compact configuration, the five planar arrays are vertically spaced with $11 \mathrm{~cm}$ gaps between each detector plane. We studied this configuration in detail in Paper II as to the effects of event cuts and reconstruction techniques on the overall sensitivity.

We have not included any active or passive shielding in our simulations. All of our background rejection relies

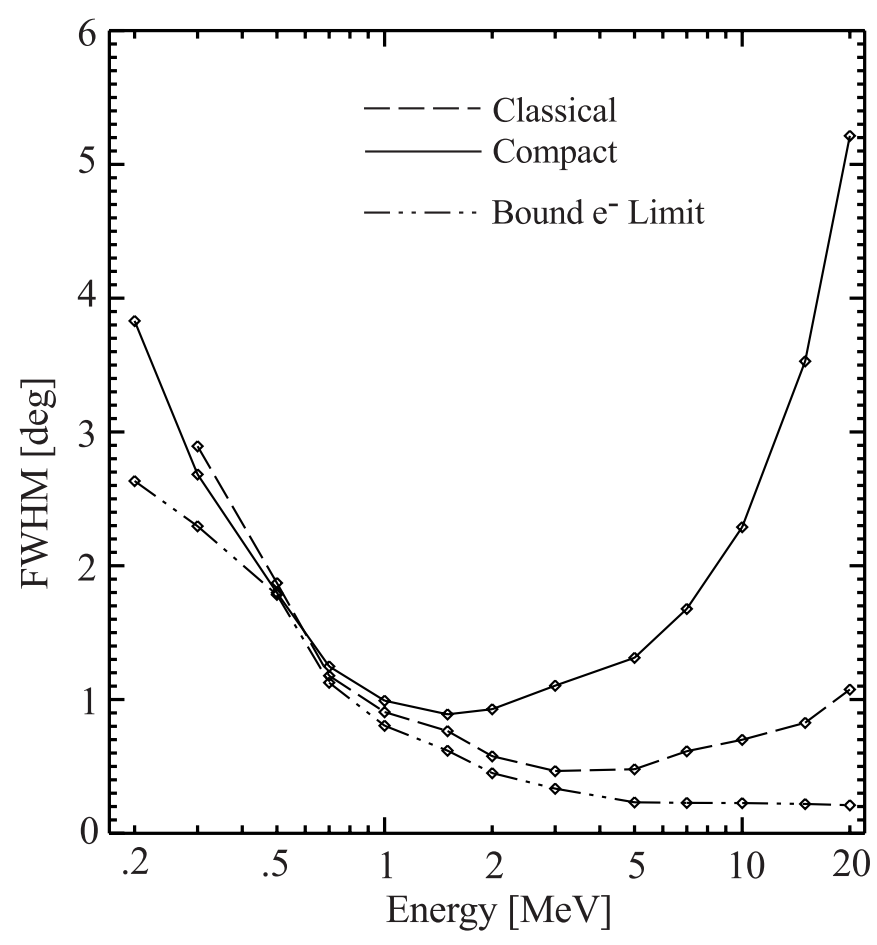

Fig. 3. Angular resolution for on-axis sources as a function of photon energy. Also shown is the limit set by Doppler broadening of bound electrons.

on the event analysis techniques presented in Paper I. The use of active shielding could potentially reduce the background and increase sensitivity. We will investigate the effects of active shielding in future work.

The two instruments were simulated using CERN's GEANT Monte Carlo code, modified to include Doppler broadening of bound electrons ${ }^{1}$. The deposited energies and positions of photon interactions are randomly modified by Gaussian distributions to reflect the assumed energy resolution (shown in Fig. 4), and $\sim 1-\mathrm{mm} F W H M$ spatial resolution. (These uncertainties were described in detail in Appendix A of Paper I.) Unresolved interactions are combined, and a detector threshold of $10 \mathrm{keV}$ is assumed.

In a high Earth orbit (HEO) environment, the components inducing soft $\gamma$-ray background in spaceborne instruments are the cosmic diffuse background (CDB) and the cosmic-ray protons that either promptly release their energy in the detector (CR prompt), or create radioactive nuclei in the instrument materials (CR delayed). The spacecraft is also a source of background events since under CR or CDB irradiation, it generates secondary particles $\left(\mathrm{p}^{+}, \mathrm{n}, \gamma\right)$ able to reach the instrument. Therefore, a numerical model of a spacecraft has been added underneath the telescopes presented in Fig. 1 in order to

\footnotetext{
1 The authors are grateful to R. M. Kippen for providing his modifications to the GEANT3 software package, which include the effects of scattering off of bound electrons. These modifications are available on-line at http://gammaray.msfc.nasa.gov/actsim/
} 
estimate a more realistic background. For convenience, we have used the detailed spacecraft model for INTEGRAL ${ }^{2}$, with the science instruments replaced by our GCT models (Lei et al. 1999). We simulate the irradiation of the GCT and the spacecraft by cosmic-ray fluxes in HEO conditions using the GEANT/GCALOR code. The simulated direction of impinging $\mathrm{CDB}$ and $\mathrm{CR}$ particles is chosen randomly according to an isotropic distribution. The CDB input spectrum is based on the recent measurements by COMPTEL (Weidenspointner 1999) and SMM (Watanabe et al. 1997). The total number of simulated CDB photons is $3 \times 10^{7}$. The solar maximum CR spectrum (Webber \& Lezniak 1974) has been used to simulate the $\mathrm{CR}$ components $\left(5 \times 10^{6}\right.$ protons were simulated $)$. This simulation provides the yields of unstable nuclei induced by CRs and their secondaries in the Ge. These yields allow us to calculate the radioactive decay rates after one year in orbit. Using these rates and the decay parameters (branching ratio, decay mode and photon cascade) of the ENSDF database, we simulate the particles $\left(\beta^{-}, \beta^{+}\right.$, $\gamma$ ) emitted by radioactive decays with GEANT in order to get the positions and the energy released when they interact in the detectors.

By studying the performance of the GCTs in a HEO, we are able to utilize the background simulations developed for SPI (Jean et al. 1997; Jean et al. 1996b), allowing us to directly compare the performance of the instruments. Simulated backgrounds in low Earth orbit (LEO) require a careful study of time variability, depending on the altitude and inclination of the orbit. The time variability of the background is expected to be lower in HEO than in LEO since in the former orbit instruments are outside of the radiation belts. However in HEO the flux of the particle inducing instrumental background can also vary (see Fig. 29 of Bouchet et al. 2001). These fluxes can be measured with a radiation monitor onboard the spacecraft (as it is the case with XMM and will be the case with INTEGRAL) giving corrections for the background subtraction in the data processing. Moreover, in redundancy with the radiation monitor, several internal background lines induced by primary and secondary CR particles interacting with the Ge detector material can be used to further estimate and correct for background variations (Naya et al. 1996). Since our purpose here is to compare the performance of various GCT configurations, a detailed analysis of the time variability of the background is beyond the scope of this paper.

\subsection{Reconstruction techniques and event cuts}

Germanium detectors pose two major complications for Compton telescope designs. Photons above $\sim 0.5 \mathrm{MeV}$ predominantly scatter multiple times in germanium before being photoabsorbed. Also, the expected event timing resolution in germanium detectors $(>10 \mathrm{~ns})$ is not adequate

${ }^{2}$ The INTEGRAL spacecraft mass model is available on-line at http://www.integral. soton.ac.uk/timm/

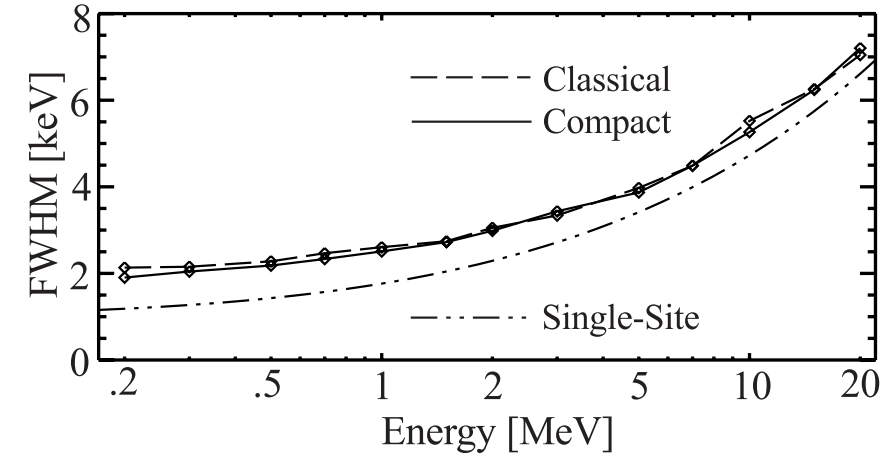

Fig. 4. Photopeak spectral resolutions, which show little dependence on the instrument configuration. For comparison is shown the assumed single-site resolution.

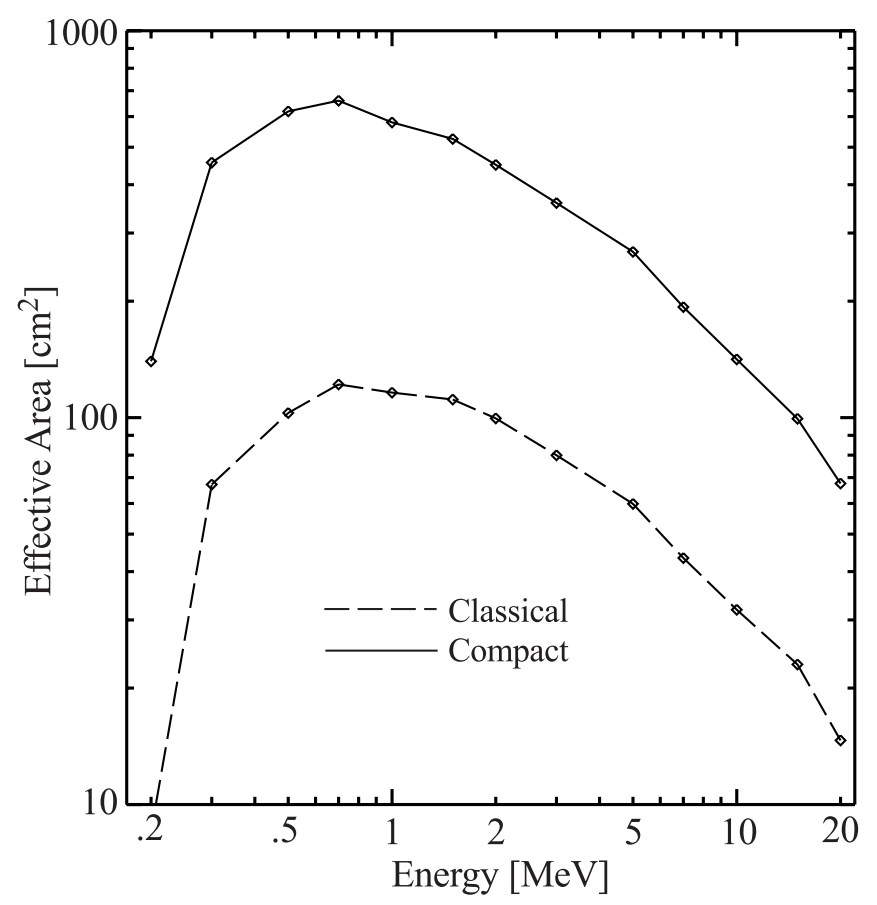

Fig. 5. On-axis photopeak effective areas, which show a strong dependence on the reconstruction technique and event cuts.

to determine the interaction order (TOF) for reasonable GCT configurations.

In Paper I, we introduced two reconstruction techniques to accurately determine the photon interaction order in GCTs, replacing the historical TOF measurement. The first technique, Compton Kinematic Discrimination (CKD), takes advantage of redundant information on the scattering angles (determined by the both the interaction locations, and the energy depositions through the Compton kinematic law) for photons which interact three or more times in the instrument $(3+$ site events $)$ to determine the most probable interaction order. CKD additionally allows efficient rejection of background events, including photons which scatter out of the instrument before fully depositing their energy (Compton continuum photons), non-localized $\beta^{-}$-decays, $\beta^{+}$-decays, and pairproduction events. The second reconstruction technique, 


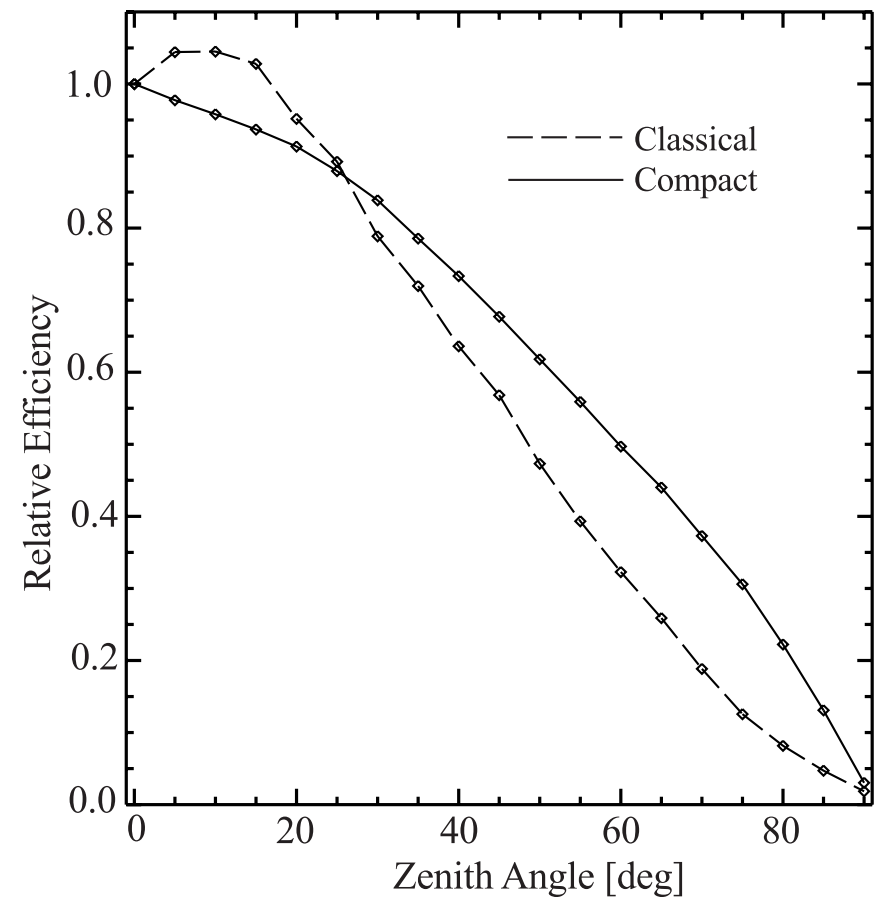

Fig. 6. Off-axis response for $1 \mathrm{MeV}$ photons relative to on-axis.

Single Scatter Discrimination (SSD), is an empirical technique which allows good determination of the interaction order for events with only two interaction sites (2-site events) for the Compact geometry, but without the benefit of background rejection. We found that below $\sim 0.5 \mathrm{MeV}$, most 2-site interactions had a unique interaction ordering that followed the Compton kinematic law, while at higher energies the ordering was not unique, but that roughly $80 \%$ of the events had their largest energy deposit in their first interaction site. As we discussed, for a given GCT configuration the performance will depend on the reconstruction techniques employed.

Telescope performance will also depend on event cuts, which can be made on the initial direction of the photon scatter, the number of interaction sites, and the minimum separation between the first and second interaction sites (minimum lever arm). The tradeoffs are generally higher efficiency at the expense of degraded angular resolution, and hence increased background. First, the uncertainty in the Compton scatter angle (angular resolution) is smaller for forward scatter events than backscatter events (Paper I, Eq. (4)). Second, 2-site events do not permit CKD background rejection, and also have a larger fraction of backscatter events than $3+$ site events. Finally, a larger minimum lever arm will minimize the effects of spatial uncertainty in the detectors, improving angular resolution and hence reducing background, but at the expense of lower efficiency. In Paper II we demonstrated that for the Classical configuration, the sensitivity was optimized by including only $3+$ site events which initially forward scattered in the instrument.

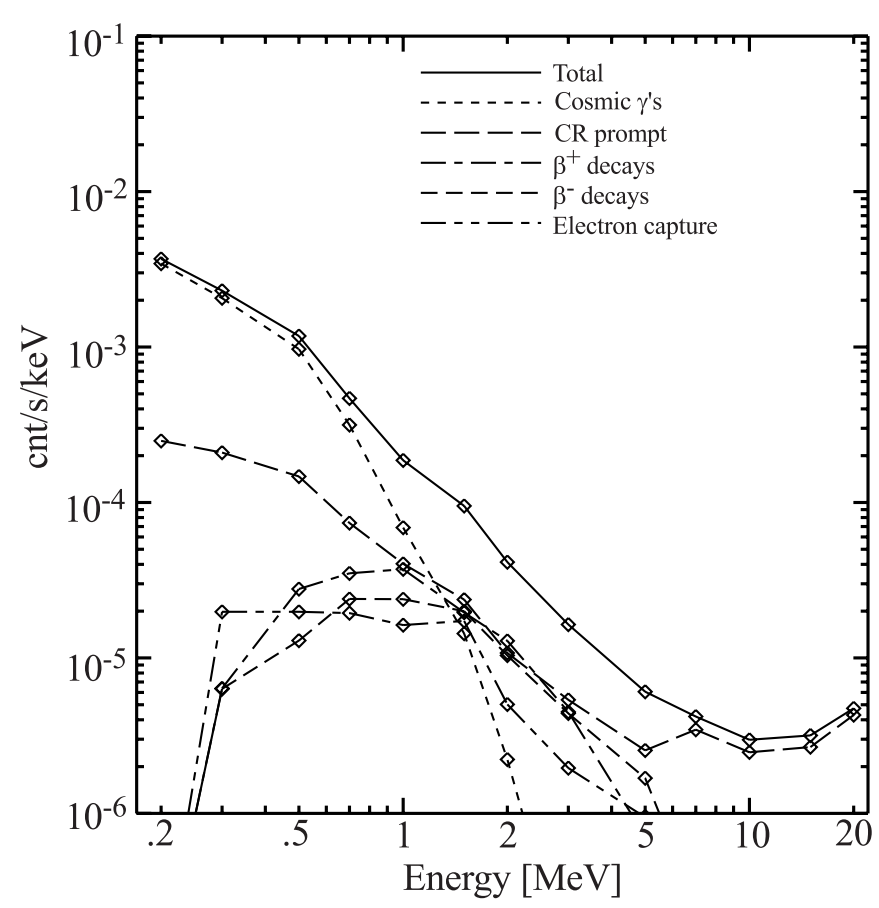

Fig. 7. Background events consistent with the $76 \%$ error circle of an on-axis point source for the Classical geometry.

\subsubsection{Classical configuration}

The performance characteristics for the Classical configuration presented in Figs. 2-10 and Table 1 were calculated using only $3+$ site events which forward scattered in the instrument, and which had their first and second scatter interactions in the upper (converter) and lower (absorber) planes respectively. This last requirement only permitted events with long lever arms $(\sim 90-100 \mathrm{~cm})$, to maximize the angular resolution. As in the case of the Compact configuration as presented in Paper II, the addition of 2-site events can increase the effective area, but at the expense of significantly higher background since CKD rejection can only be used on $3+$ site events. For example, at $1 \mathrm{MeV}$ the inclusion of 2-site events in the Classical configuration increases the effective area by a factor of 1.1 and the background by a factor of 2.9 , for an overall factor 1.6 degradation in the sensitivity. The 2 -site events are also more complicated for the Classical geometry than the Compact geometry. Above $0.5 \mathrm{MeV}$, a unique interaction order can not be determined by the energy depositions alone. For the Compact geometry we demonstrated that $\sim 80 \%$ of the 2-site photopeak events had their largest energy deposit in their first interaction site, which could be empirically used to determine the interaction order. For the Classical geometry, $\sim 50 \%$ of 2 -site photopeak events have their largest energy deposit in the first interaction site, and $\sim 60 \%$ forward scatter in the instrument. These empirical numbers indicate that the interaction order for 2-site events above $0.5 \mathrm{MeV}$ in the Classical geometry is statistically ambiguous. 


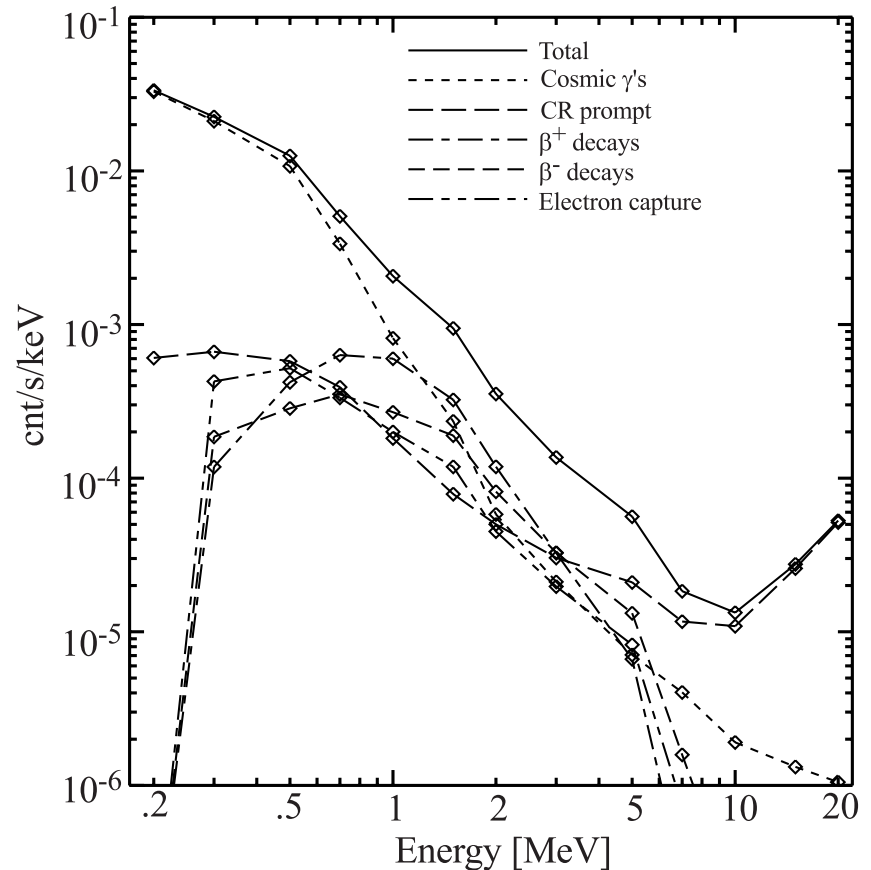

Fig. 8. Background events consistent with the $76 \%$ error circle of an on-axis point source for the Compact geometry.

\subsubsection{Compact configuration}

In Paper II, we demonstrated how the use of CKD and allowing only $3+$ site events which initially forward scatter in the instrument (Case 1 in Paper II) maximized the sensitivity for the Compact geometry. The inclusion of 2-site events and backscatter events (Case 2 in Paper II) increased the effective area at $1 \mathrm{MeV}$ by a factor of 1.3 , and the background by a factor of 3.4, for an overall factor of 1.4 degradation in sensitivity. The performance results presented in Figs. 2-10 and Table 1 were calculated using only $3+$ site events which forward scattered in the instrument, and which have their first and second interactions in separate detector planes.

\section{Performances calculations}

The performance of a Compton telescope is not simple to characterize due to the unknown direction of the Compton recoil electron, providing an annulus on the sky instead of a point for the impinging photon direction (see Fig. 1). The background calculations and sensitivity require special care in defining. Moreover, imaging techniques for Compton telescope are complex and require heavy data processing (e.g., Maximum Likelihood method, Maximum Entropy, etc.). Since our purpose is to estimate and optimize the performance of a GCT we use simple methods for determining performance. Here we attempt to explain in detail how the various performance characteristics were defined and calculated for these simulations.
Table 1. Performance at $1 \mathrm{MeV}$.

\begin{tabular}{lcc}
\hline Performance & Classical & Compact \\
\hline Effective Area $\left[\mathrm{cm}^{2}\right]$ & 118 & 590 \\
ARM $F W H M[\mathrm{deg}]$ & 0.81 & 0.96 \\
$\Delta E F W H M[\mathrm{keV}]$ & 2.49 & 2.50 \\
FOV $[\mathrm{str}]$ & 2.5 & 3.2 \\
Narrow Line Sensitivity & 8.5 & 5.4 \\
{$\left[10^{-7} \mathrm{ph} / \mathrm{cm}^{2} / \mathrm{s}\right]$} & & \\
Continuum Sensitivity & 14.0 & 9.1 \\
{$\left[10^{-6} \mathrm{ph} / \mathrm{cm}^{2} / \mathrm{s} / \mathrm{MeV}\right]$} & & \\
\hline
\end{tabular}

\subsection{Angular resolution}

Angular resolution in Compton telescopes is often described in terms of the angular resolution measure (ARM), defined as the difference between the initial photon scatter angle in the instrument and the scatter angle reconstructed from the Compton scatter formula. The ARM distributions of $1.0 \mathrm{MeV}$ photons from an on-axis source are shown in Fig. 2 for the Classical and Compact configurations. Both ARM distributions are highly non-Gaussian, with sharp central peaks and broad wings. The shape of the ARM distributions is dominated by the momentum distribution of bound electrons in Ge atoms. The wings are relatively higher for the Compact configuration because the effects of Doppler broadening are stronger for larger scatter angles. The wings also tend to be less significant at higher energies for both configurations. The $F W H M$ of the on-axis ARM distribution as a function of energy is shown in Fig. 3, as well as the limits imposed by Doppler broadening of bound electrons. This broadening dominates the angular resolution below $\sim 1 \mathrm{MeV}$, so that the two configurations have similar angular resolutions. At higher energies, the angular resolution is dominated by the degradation in the spatial resolution due to the increased recoil-electron range, so the Classical configuration, with its relatively long lever arm, maintains better angular resolution than the Compact configuration.

Since the ARM distributions are highly non-Gaussian, it is necessary to define the effective FWHM in addition to the $F W H M$. The effective $F W H M$ is defined as the width of the ARM distribution which contains $76 \%$ of the photon events. For Gaussian distributions these two widths are equal. For our ARM distributions, the effective $F W H M$ tends to be $2-3$ times broader than the $F W H M$. For the background and sensitivity calculations below, we included all event circles consistent with the effective FWHM of the source location.

\subsection{Spectral resolution}

The photopeak $F W H M$ energy resolution is broader than the single-site resolution due to the addition in quadrature of electronic noise for multiple interaction sites. 
The resulting lines remain Gaussian, however. The photopeak FWHM energy resolutions are shown in Fig. 4, with the assumed single-site resolution shown for comparison. The photopeak resolution is nearly identical for both configurations. At $1 \mathrm{MeV}$, the photopeak FWHM corresponds to a $\sim 33 \%$ broadening over the single-site resolution (1.77 keV to $2.36 \mathrm{keV}$ ).

\subsection{Effective area}

The on-axis photopeak effective areas as a function of energy are shown in Fig. 5, determined by integrating over the ARM distributions. The Compact geometry has factors $\geq 5$ larger effective area than the Classical configuration. For both configurations, the effective area is maximum around $0.7 \mathrm{MeV}$. The Compact configuration maintains a significant efficiency down to $0.2 \mathrm{MeV}$, while the Classical configuration has a steep drop in effective area below $0.3 \mathrm{MeV}$. This drop is due to both more isotropic photon scattering at lower energies, and because low energy photons that scatter in the first detection plane must have a small scattering angle in order to be detected in the absorber (or else they escape the telescope), in which case they release energy below the $10 \mathrm{keV}$ threshold in the converter and are not detected. In the Compact configuration low energy photons do not require a small angle scatter to be detected in the absorber. At higher energies, the effective areas decrease slowly with energy at similar rates for the two configurations.

\subsection{Field of view}

The angular response defined relative to the on-axis response is shown in Fig. 6 for $1 \mathrm{MeV}$ photons. Since the instrument designs are not radially-symmetric, the angular response can be expected to vary depending on the azimuthal angle of the source. Here we calculated the response at an azimuth angle of $22.5^{\circ}$ relative to the $x$-axis as defined in Fig. 1. This should represent the mean angular response of the instruments. The fact that the Classical response peaks off-axis is not surprising. The peak scatter angle for $1 \mathrm{MeV}$ photons is $\sim 30^{\circ}$, so off-axis photons can be expected to have a higher probability of scattering into the solid angle of the absorber layers. For the Compact geometry where the absorbing planes subtend a much larger solid angle, this effect is less important. The field-of-view (FOV), defined as the angular response integrated over all solid angles, is given in Table 1 for both configurations.

\subsection{Backgrounds}

The total backgrounds for on-axis point source observations are shown in Fig. 7 for the Classical and Fig. 8 for the Compact configurations. These backgrounds are defined as all events whose event circles are consistent with a photon originating from within the $76 \%$ error circle (effective $F W H M$ ) of the energy-dependent ARM distribution

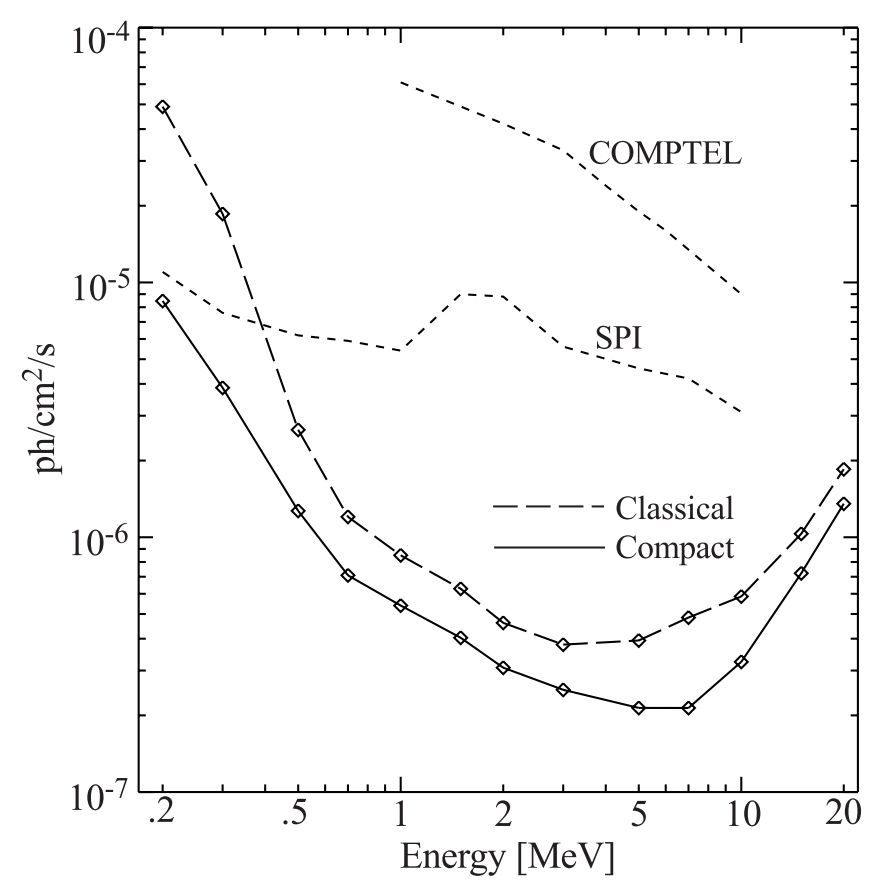

Fig. 9. The narrow-line sensitivity $\left(3 \sigma, 10^{6} \mathrm{~s}\right)$, for an onaxis point source. Shown for comparison are the narrowline sensitivities of SPI (Jean et al. 2000) and COMPTEL (Schönfelder et al. 1993).

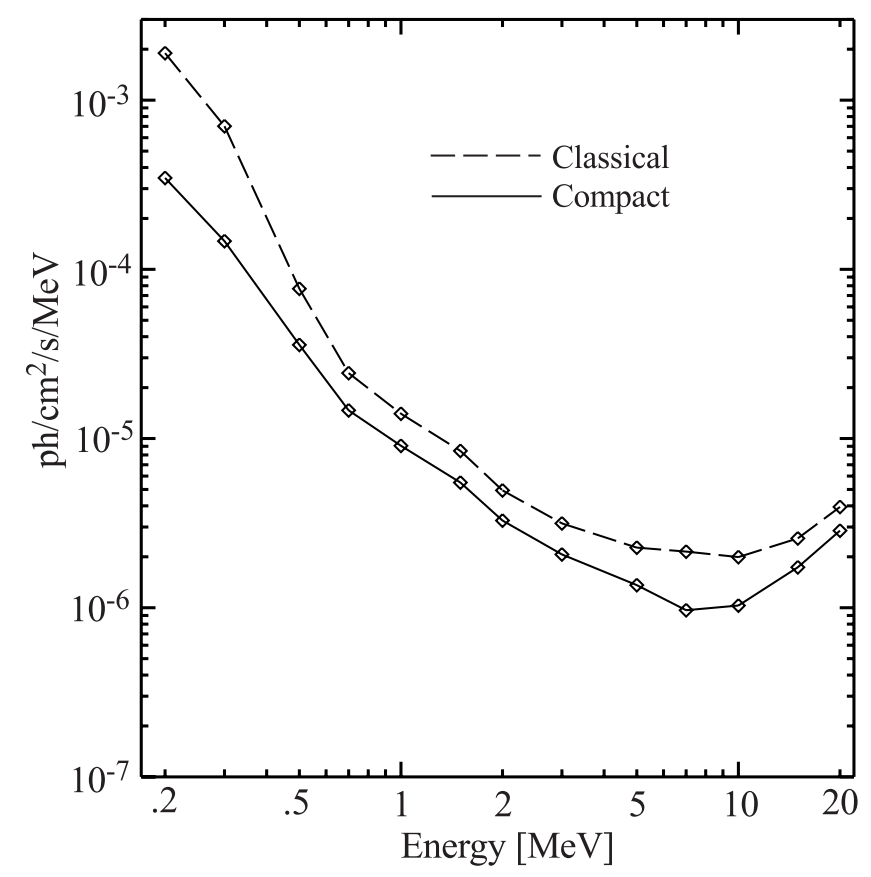

Fig. 10. The continuum sensitivity $\left(3 \sigma, 10^{6} \mathrm{~s}, \Delta E=E\right)$, for an on-axis point source.

for each geometry. Events consistent with the source location, which were not rejected by any of the background rejection techniques, were combined into the spectra in Figs. 7 and 8. The Classical configuration has a factor of 10 lower background at $1 \mathrm{MeV}$ than the Compact configuration due to its combination of lower effective area, slightly higher angular resolution, and smaller FOV. 


\subsection{Narrow line sensitivity}

The $3 \sigma$ narrow line sensitivity for an on-axis source was calculated using the formula

$F_{3 \sigma}=\frac{3 \sqrt{B \Delta E}}{(0.76)^{2} A_{\mathrm{eff}} \sqrt{T}}$,

where $B$ is the energy-dependent background continuum shown in Figs. 7 and 8, $\Delta E$ is the spectral resolution presented in Fig. $4, A_{\text {eff }}$ is the effective area shown in Fig. 5; and $T$ is the observing time. The factor $(0.76)^{-2}$ comes from the fact that we are including only source photons within the $F W H M$ of the photopeak, and the effective $F W H M$ of the ARM distribution. (The background calculations also assumed this same effective $F W H M$ of the ARM distribution). This formula also assumes that the background at the source location is estimated at multiple off-source locations in the instrument FOV; otherwise, in the case of an off-source background observation there would be an additional factor of $\sqrt{2}$ in the numerator. We have not accounted for instrument deadtimes when processing photon or charged-particle events; however, we estimate that deadtime will affect the sensitivity by $\ll 5 \%$. The calculated narrow-line sensitivities are shown in Fig. 9 for a $10^{6} \mathrm{~s}$ observation. We see that the increase in effective area of the Compact configuration improves the sensitivity relative to the Classical configuration at all energies.

\subsection{Continuum sensitivity}

The $3 \sigma$ continuum sensitivity for an on-axis source at energy $E$ was calculated using the formula

$$
S_{3 \sigma}=\frac{3 \sqrt{B}}{(0.76) A_{\mathrm{eff}} \sqrt{E T}},
$$

where we have once again assumed that the background at the source location is estimated at multiple off-source locations in the FOV. This formula also assumes an energy band equal in width to the mean energy of the band $(\Delta E=E)$, for comparison with the published continuum sensitivity curves for most other instruments. Only one factor of $(0.76)^{-1}$ appears because we are including all events within an energy band, not a fraction of the photopeak. The calculated continuum sensitivities are shown in Fig. 10 for a $10^{6} \mathrm{~s}$ observation. Once again the Compact configuration is significantly more sensitive at all energies than the Classical configuration.

\section{Comparison}

The Classical configuration was originally proposed as a means of utilizing the very high spectral resolution of GeDs in order to achieve angular resolutions $\ll 1^{\circ} \quad$ (Johnson et al. 1996; Jean et al. 1996a; Boggs 1998). These earlier estimates of angular resolution, however, did not account for the limits set by Doppler broadening due to Compton scattering off of bound electrons. This broadening dominates the angular resolution for photon energies below $\sim 2 \mathrm{MeV}$, so that the advantage of the long lever arm is only significant at higher energies. At $1 \mathrm{MeV}$, the angular resolution of the Compact geometry is degraded relative to the Classical by a factor of 1.2. The degradation becomes more significant at higher energies where the Doppler broadening of bound electrons is less significant than the spatial uncertainty in the interaction locations.

The closer spacing of the the detector planes in the Compact geometry, and the use of each plane as a "converter" as well as "absorber," leads to factors of $\geq 5$ improvements in overall effective area relative to the Classical Compton telescope geometry. This increase in effective area ultimately provides improved sensitivity. The Compact geometry also maintains better performance to lower energy photons $(\leq 0.5 \mathrm{MeV})$.

In addition, the Compact geometry has a $30 \%$ larger FOV than the Classical configuration. This factor leads to an effective increase in sensitivity because a given source will be visible in the Compact telescope FOV for longer times than in the Classical. Scaling the sensitivities by the FOV, the Compact configuration is a full factor of 2.0 more sensitive to both narrow line and continuum sources.

\section{Conclusion}

Their high spectral resolution, good efficiency, large FOV, and excellent background rejection capabilities make GCTs an attractive option for an Advanced Compton Telescope following INTEGRAL. The Compact GCT presented here would provide an order of magnitude improvement in nuclear line sensitivity over INTEGRAL/SPI, allowing the pursuit of new scientific goals such as the systematic study of type Ia $\mathrm{SNe}$, as well as improved sensitivity to the positron annihilation line $(0.511 \mathrm{MeV})$, ${ }^{26} \mathrm{Al}(1.809 \mathrm{MeV}),{ }^{60} \mathrm{Fe}(1.173,1.332 \mathrm{MeV})$, and ${ }^{44} \mathrm{Ti}$ (1.157 MeV). Moreover, imaging capabilities of the GCT will allow improved mapping of these line emissions in our Galaxy, giving valuable insights on the origin and propagation of the positrons, the sites and physics of Galactic nucleosynthesis, and the locations of the most recent Galactic supernovae. The GCT will also provide a similar improvement in continuum sensitivity for the study of compact sources at $\mathrm{MeV}$ energies and of diffuse galactic emission.

In the search for prompt emission from supernovae, the sensitivity is degraded relative to the narrow line sensitivity when the observed line is broader than the spectral resolution, due to the integration of more background counts in the line. The Compact GCT has a sensitivity of $1.9 \times 10^{-6} \mathrm{ph} / \mathrm{cm}^{2} / \mathrm{s}$ to $28 \mathrm{keV}\left(10000 \mathrm{~km} \mathrm{~s}^{-1}\right)$ FWHM broad ${ }^{56} \mathrm{Co}(0.847 \mathrm{MeV})$ lines, corresponding to the expected peak flux from a type Ia supernova at $40 \mathrm{Mpc}$ (Burrows \& The 1990; Chan \& Lingenfelter 1991). Within this distance, the expected SN Ia rate is $5-$ 10 per year (Cappellaro et al. 1997). The spectral resolution of the GCT would resolve this ${ }^{56} \mathrm{Co}$ line at $850 \mathrm{~km} \mathrm{~s}^{-1}$ 
$F W H M$, allowing identification of the explosion mechanism of SN Ia (Gómez-Gomar et al. 1998).

Sensitivity of GCTs can be further improved by using an anticoincidence system with active shield around the GCT in order to reduce background due to upward scattered photons from the spacecraft, as well as internal radioactive decays with two or more de-excitation photons. Moreover, such a shield would reject source photons that do not release the totality of their energy in the GCT, reducing the Compton continuum.

We can also imagine making a tunable GCT by mechanically adjusting the distance between detector planes in order to get an angular resolution adapted to the scientific objectives.

In this study, GCT sensitivities have been estimated in HEO condition, nevertheless we can expect a background reduction of $\sim 2$ and therefore a improvement in sensitivities of $\sim 40 \%$ in low Earth orbit. Unless the orbit has a low inclination angle, however, the instrument will be periodically irradiated by South Atlantic Anomaly particles and influenced by (or subject to) atmospheric gamma-emission inducing variability in the background as a function of time and pointing direction with respect to the Earth.

Acknowledgements. S. Boggs acknowledges the California Space Institute and NASA Grant NAG5-5285 for support. Thanks to G. Weidenspointner and J. Kurfess for useful discussions, and to the anonymous referee for a number of useful suggestions.

\section{References}

Aprile, E., Bolotnikov, A., Chen, D., \& Mukherjee, R. 1993, Nucl. Inst. Meth. A, 327, 216

Aprile, E., et al. 2000, Proc. 5th Compton Symp., AIP Conf. Proc., 510, 799

Boggs, S. E., \& Jean, P. 2001, Proc. 4th INTEGRAL Workshop (Alicante, Spain), in press, Paper II

Boggs, S. E., \& Jean, P. 2000, A\&AS, 145, 311, Paper I
Boggs, S. E. 1998, Ph.D. Dissertation (University of California, Berkeley)

Bouchet, L., et al. 2001, ApJ, 548, 990

Burrows, A., \& The, L.-S. 1990, ApJ, 360, 626

Cappellaro, E., et al. 1997, A\&A, 322, 431

Chan, K. W., \& Lingenfelter, R. E. 1991, ApJ, 368, 515

Gómez-Gomar, J., Isern J., \& Jean, P. 1998, MNRAS, 295, 1

Herzo, D., et al. 1975, Nucl. Inst. Meth., 123, 583

Jean, P., Naya, J. E., Olive, J. F., \& von Ballmoos, P. 1996a, A\&AS, 120, 673

Jean, P., et al. 1996b, SPIE, 2806, 457

Jean, P., et al. 1997, Proc. 2nd INTEGRAL Workshop, ESA SP-382, 635

Jean, P., Vedrenne, G., Schönfelder, V., et al. 2000, Proc. 5th Compton Symp., AIP Conf. Proc., 510, 708

Johnson, W. N., et al. 1993, ApS, 86, 693

Johnson, W. N., et al. 1996, SPIE, 2518, 74

Kroeger, R. A., et al. 2000, IEEE Nucl. Sci. Symp. Conf. Proc., in press

Lei, F., et al. 1999, Proc. 3rd INTEGRAL Workshop, Astro. Lett. Comm., 39, 373

Lockwood, J. A., Hsieh, L., Friling, L., Chen, C., \& Swartz D. 1979, JGR, 84, 1402

Naya, J. E., et al. 1996, Nucl. Inst. Meth. A, 368, 832

O'Neill, T. J., et al. 2000, Proc. 5th Compton Symp., AIP Conf. Proc., 510, 804

Schönfelder, V., Hirner, A., \& Schneider, K. 1973, Nucl. Inst. Meth., 107, 385

Schönfelder, V., et al. 1993, ApS, 86, 657

Schopper, F., et al. 2000, IEEE Nucl. Sci. Symp. Conf. Proc., in press

Ubertini, R., et al. 2000, Proc. 5th Compton Symp., AIP Conf. Proc., 510, 684

von Ballmoos, P., Diehl, R., \& Schönfelder, V. 1989, A\&A, 221, 396

Watanabe, K., et al. 1997, in Proc. of the 4th Compton Symp., ed. C. D. Dermer, M. S. Strickman, \& J. D. Kurfess, AIP Conf. Proc., 4101, 223

Webber, W. R., \& Lezniak, J. A. 1974, Astrophys. Space Sci., 30, 361

Weidenspointner, G. 1999, Ph.D. Dissertation, Technical (University Munich, Germany) 